Hernández Ramos, J.P. \& Martínez Abad, F. (2021). La importancia de la actitud del docente universitario: validación de una escala para su consideración. Revista Electrónica Interuniversitaria de Formación del Profesorado, 24(1), 59-71.

DOI: https://doi.org/10.6018/reifop.414781

\title{
La importancia de la actitud del docente universitario: validación de una escala para su consideración
}

\author{
Juan Pablo Hernández Ramos, Fernando Martínez Abad \\ Universidad de Salamanca
}

\section{Resumen}

El estudio de la calidad y la mejora de la docencia universitaria se basa en la mayoría de las ocasiones en la formación del docente, dejando a un lado otros factores transcendentales como puede ser la actitud. Considerada como el comportamiento del docente en base a sus conocimientos y experiencias, este trabajo presenta la validación de una escala de actitud hacia la docencia en base a 3 dimensiones: personal, docente y profesional. Tras una validación de contenido por jueces, se aplica la escala a una muestra, representativa por rama de conocimiento y categoría profesional, de 161 profesores universitarios de la Universidad de Salamanca. El análisis psicométrico de la escala, formada por 13 ítems tipo Likert con cinco opciones de respuesta, muestra correlaciones ítem total medias-bajas, un índice de fiabilidad aceptable ( $\alpha$ de Cronbach=.75) y una estructura factorial clara y consistente donde se confirma la existencia de los tres factores. Así mismo, se destaca la mayor distinción de las dimensiones docente (.854) y profesional (.885), frente a la investigadora (.553). Se concluye incidiendo en la importancia de considerar las actitudes del profesorado como indicador relevante de estudio en la enseñanza superior, empleando escalas válidas y fiables como la que se ofrece en este artículo. Como líneas de investigación futuras, queda abierta la puerta a una nueva validación del instrumento en nuevos contextos de enseñanza universitaria.

\section{Palabras clave}

Psicometría; Actitud del docente; Enseñanza Superior; Ciencias Sociales. 


\title{
Importance of the attitude of the university teacher: validation of a scale for consideration
}

\begin{abstract}
The study of the quality and improvement of university teaching is mostly based on teacher training, leaving aside other transcendental factors such as attitude. Considered as the behavior of the teacher based on their knowledge and experiences, this work shows the validation of a scale of attitude towards teaching based on 3 dimensions: personal, teaching and research. After content validation by judges, the scale is applied to a representative sample, by branch of knowledge and professional category, of 161 university teachers at the University of Salamanca. The psychometric analysis of the scale, made up of 13 Likert type items with five answer possibilities, shows correlations between average and low total items, an acceptable reliability index ( $\alpha$ de Cronbach=.75) and a clear and consistent factor structure where the existence of the three factors is confirmed. In addition, there is a greater distinction between the teaching (.854) and professional (.885) dimensions, as opposed to the researcher $(.553)$. It concludes by stressing the importance of considering the attitudes of teachers as a relevant indicator of study in higher education, using valid and reliable scales such as that offered in this article. As future lines of research, the door remains open to a new validation of the instrument in new contexts of higher education.
\end{abstract}

\section{Key words}

Psychometrics; Teacher attitudes; Higher education; Social sciences.

\section{Introducción}

En el ámbito universitario, desde el inicio del siglo XXI se han desarrollado infinidad de programas en post de la innovación y la mejora educativa. Además de los cambios estructurales acaecidos en la enseñanza superior, se han producido modificaciones importantes en los procesos de enseñanza-aprendizaje (Cabero et al., 2018; Cáceres \& Salas, 2012; Jiménez et al., 2019). Una nueva obligación instructiva que repercute en los docentes con la necesidad de una formación pedagógica diferente a efectos de prepararse para estimular la innovación en sentido crítico y la creatividad; siendo estériles las prácticas metodológicas basadas en el simple hecho de trasmitir los conocimientos teóricos de la disciplina.

Ciertos autores (Cabero et al., 2018; Gutiérrez et al., 2018; Mercado et al., 2016) coinciden en considerar los cambios estructurales, como la oportunidad idónea para mejorar la enseñanza universitaria en base a ofrecer a los estudiantes una formación de calidad que atienda sus necesidades. Sin embargo, estas permutas organizativas y formativas le han llegado al docente universitario de forma vertical, de manera que no han sido consideradas sus aptitudes y actitudes. $Y$ en este sentido tan importante es el desarrollo de buenos planes de formación para el profesorado, como la consideración de sus opiniones y creencias. Entendiendo la actitud como la predisposición a proceder de una forma determinada en base a componentes cognitivos, afectivos y conductuales, en donde todos los elementos están interrelacionados (Erwin, 2001), surgen estudios centrados en conocer y analizar la actitud del profesorado hacia factores relacionados con la docencia (Aguilar \& Cortés, 2016; Berkant 
\& Derer, 2016; Burgueño et al., 2018; Çakmak, 2011; Covarrubias \& Mendoza, 2016; FernándezBatanero \& González, 2015; Márquez \& Madueño, 2016). De cara al buen desarrollo de este tipo de estudios, se considera necesaria la existencia de instrumentos de medida válidos, diseñados bajo criterios científicos.

Para garantizar la fiabilidad y validez de estos instrumentos surgen, en el contexto nacional e internacional, multitud de investigaciones que estudian las propiedades psicométricas de instrumentos diseñados al efecto (Caldera et al., 2018; De Haro et al.,2020; Hernández-Ramos et al., 2014, 2016; Hurtado \& Madueño, 2017; Martínez-Clares \& González-Lorente, 2019; Martinez-León et al., 2018; Mercado-Salgado et al., 2016; Sicilia et al., 2017; Zych et al., 2018).

A pesar de la existencia de gran cantidad de escalas de actitud diseñadas y validadas para su aplicación a docentes, en muchos casos están centradas en niveles de enseñanza básicos (Burgueño et al., 2018; Maier et al., 2013; Ozturk, 2010; Toraman et al., 2015), abordan ámbitos docentes específicos (Muñoz-Cantero et al., 2019; Rodríguez-Conde et al., 2012; Sales et al., 2006; Zych et al., 2018) o muestran carencias en el proceso de validación: pilotajes a partir de muestras muy pequeñas, valores de fiabilidad bajos (Ozturk, 2010), análisis exploratorios poco reveladores o falta de análisis confirmatorios que reafirmen la estructura factorial (Chen, 2006; Naya et al., 2014; Toraman et al., 2015). Como consecuencia, no se ha podido detectar en la literatura científica actual, ninguna escala para la evaluación de actitudes hacia la docencia del profesorado universitario y con una validación psicométrica adecuada.

En este sentido, a pesar de que los ítems y dimensiones conformantes de los diferentes instrumentos analizados se podrían considerar para elaborar una escala genérica sobre actitudes hacia la docencia, debido a su naturaleza concreta y a las debilidades psicométricas localizadas, surgen dudas sobre su solvencia para un fin más global como el que se busca.

Como respuesta a estas cuestiones, la presente investigación plantea el objetivo de (re)validar, en la actualidad, la Escala de actitud del profesorado universitario hacia la docencia, diseñada por el Grupo Helmántica (1999) hace más de 20 años. El reto consiste, por tanto, en comprobar si, tras los cambios acontecidos durante los últimos años en las universidades, las actitudes y creencias del profesorado hacia la docencia siguen configurándose en torno al mismo constructo definido en un contexto universitario radicalmente distinto al actual.

\section{Metodología}

La metodología de la investigación se elabora en base al objetivo planteado. Se desarrolla un proceso sistemático, controlado, empírico y objetivo, con la finalidad de aportar explicaciones aceptables a la realidad mostrada.

La Escala de Actitud hacia la Docencia Universitaria, recogida en la figura 1, está conformada por 13 ítems formulados a partir de una escala tipo Likert con las siguientes opciones de respuesta: totalmente en desacuerdo (1), en desacuerdo (2), Ni acuerdo ni desacuerdo (3), de acuerdo (4) y Totalmente de acuerdo (5); y aborda la actitud del profesorado universitario hacia su figura como docente en base a 3 dimensiones teóricas: actitud hacia la docencia en base al desarrollo personal, actitud hacia la docencia en base al desarrollo como docente y actitud hacia la docencia en base al desarrollo como profesional e investigador.

\section{Participantes}

La población diana establecida para el presente estudio es el profesorado universitario. Dado el carácter de pilotaje, se cree conveniente escoger una muestra de profesorado proveniente de una universidad que oferte un gran número de titulaciones repartidas entre las ramas de 
conocimiento y que reciba estudiantes geográficamente diversos, como la Universidad de Salamanca. De sus 2329 profesores, se obtiene una muestra no probabilística de 161 sujetos. Se comprueba la representatividad proporcional de la muestra con respecto a la población en base a la rama de conocimiento y la categoría profesional de los docentes. Para ello, se aplica una prueba de bondad de ajuste basada en el estadístico Chi Cuadrado; y los resultados indican que la muestra tomada se distribuye conforme a la población, tanto en función de la rama de conocimiento $(\chi 2=0.843 ; p=.933)$, como la categoría profesional $(\chi 2=1.091 ; p=.857)$.

\section{ESCALA DE ACTITUD HACIA LA DOCENCIA UNIVERSITARIA}

\begin{tabular}{|c|c|c|}
\hline \multirow{4}{*}{ 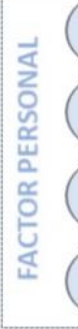 } & Me gusta enseñar en la Universidad. & $1-2-3-4-5$ \\
\hline & Ser profesor universitario te permite realizar un trabajo creativo. & $1-2-3-4-5$ \\
\hline & $\begin{array}{l}\text { Un aspecto positivo de la Docencia Universitaria es la independencia } \\
\text { que se posee. }\end{array}$ & $1-2-3-4-5$ \\
\hline & $\begin{array}{l}\text { El contacto con los estudiantes es un hecho enriquecedor para el } \\
\text { profesor universitario. }\end{array}$ & $1-2-3-4-5$ \\
\hline \multirow{5}{*}{ 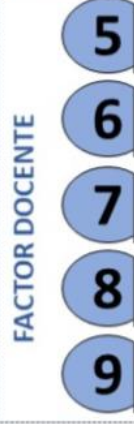 } & $\begin{array}{l}\text { Los profesores universitarios deben tener una buena formación } \\
\text { pedagógica. }\end{array}$ & $1-2-3-4-5$ \\
\hline & El profesor debe ser algo más que un puro transmisor de información. & $1-2-3-4-5$ \\
\hline & $\begin{array}{l}\text { La preparación de las clases es un trabajo muy importante para el } \\
\text { buen desarrollo de estas. }\end{array}$ & $1-2-3-4-5$ \\
\hline & $\begin{array}{l}\text { El sistema mediante el cual se evalúa a los estudiantes es uno de los } \\
\text { temas que merece mayor atención por parte del profesor. }\end{array}$ & $1-2-3-4-5$ \\
\hline & $\begin{array}{l}\text { El buen profesor universitario se preocupa por ofrecer a sus } \\
\text { estudiantes explicaciones claras que puedan comprender. }\end{array}$ & $1-2-3-4-5$ \\
\hline \multirow{4}{*}{ 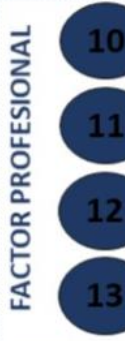 } & $\begin{array}{l}\text { El profesor universitario debería preocuparse más de lo que se } \\
\text { preocupa actualmente por la docencia. }\end{array}$ & $1-2-3-4-5$ \\
\hline & La enseñanza es la función clave del profesor Universitario. & $1-2-3-4-5$ \\
\hline & $\begin{array}{l}\text { La labor desarrollada con mis estudiantes da sentido a mi trabajo } \\
\text { profesional. }\end{array}$ & $1-2-3-4-5$ \\
\hline & $\begin{array}{l}\text { Me preocupo porque los estudiantes obtengan buenos resultados } \\
\text { académicos. }\end{array}$ & $1-2-3-4-5$ \\
\hline
\end{tabular}

Figura 1. Escala de actitud hacia la docencia

Teniendo en cuenta dichas similitudes, en lo referente a la rama de conocimiento, la muestra la forman 36 profesores de Arte y Humanidades, 27 de Ciencias, 50 de Ciencias de la Salud, 39 de Ciencias Sociales y 9 de Ingeniería y Arquitectura. En función de la categoría profesional colaboran en el estudio 18 catedráticos, 76 profesores funcionarios (PTU y CEU), 21 contratados a tiempo completo (PCD y PAD) y 46 a tiempo parcial (asociados, colaboradores y becarios de investigación.

\section{Variables y procedimiento}

Se presenta la revalidación de la Escala de Actitud hacia la Docencia Universitaria, compuesta de 13 ítems medidos a través de una escala de respuesta tipo Likert y 3 dimensiones teóricas actitudinales (en base al desarrollo personal, al desarrollo como docente y al desarrollo profesional). 
Dado que la validación de un instrumento no es un proceso acabado sino permanente que conlleva continuas comprobaciones empíricas (Soriano, 2014; Zych et al., 2018), a pesar de que la escala ya ha sido empleada en otros estudios (Hernández-Ramos et al., 2017), debido al paso del tiempo y a los cambios acontecidos en el contexto universitario, el primer paso a ejecutar se centró en un proceso de revalidación de contenido mediante una evaluación de jueces (10 expertos, todos ellos profesores universitarios, concretamente dos docentes por cada una de las cinco ramas de conocimiento). Los diez evaluadores seleccionados completaron la validación de manera anónima e independiente a través de una encuesta online. En la misma valoraron, en una escala de 1 a 5 puntos (1=Ninguna, 2=Alguna, 3=Bastante, 4=Mucha y 5=Total), la validez, claridad y relevancia de la escala. Por otro lado, valoraron de manera cualitativa los posibles problemas detectados relacionados con los ítems (cuestiones técnicas, ambigüedades, comprensión, etc.), aportando sugerencias de mejora para cada ítem en el caso de estimarlo oportuno. Una vez finalizado el proceso de validación de contenido, el cuestionario fue enviado a través de medios electrónicos al profesorado y resuelto por los mismos vía online, a través de la tecnología Google Drive.

Tras la obtención, procesamiento y ordenación de los datos de la muestra piloto, se realizó el estudio de las propiedades psicométricas del instrumento presentado. Las dos características principales de un instrumento de medición son la fiabilidad y la validez (Eiroa et al., 2008; Kerlinger \& Lee, 2002; Prieto, 2012; Urbano-Contreras et al., 2019; Zych et al., 2018). La fiabilidad se refiere a que un objeto de estudio medido en varias ocasiones con el mismo instrumento siempre ofrecerá los mismos resultados, mientras que la validez, tradicionalmente se ha considerado que el instrumento es válido si mide el constructo que pretende medir. No obstante, se debe considerar que la validez no es una propiedad intrínseca de los instrumentos, sino que depende de diferentes factores como la población o el contexto de aplicación (Soriano, 2014).

Para valorar el grado de relación entre cada uno de los 13 ítems en la escala y en sus dimensiones, se analizaron los índices de correlación ítem-total. Posteriormente, para el estudio de fiabilidad se calculó el coeficiente de a de Cronbach, que pone a prueba el grado de consistencia interna de la escala al establecer una relación entre los reactivos y la puntuación total del instrumento (Abad, 2011; Prieto, 2012).

En cuanto a la validez factorial, una vez comprobadas las condiciones previas necesarias para la aplicación del análisis factorial, se aplicó en primer lugar un Análisis Factorial Exploratorio (AFE) a través de los componentes principales y en segundo un Análisis Factorial Confirmatorio (AFC). Cabe destacar que el AFE no es suficiente para garantizar la validez de una escala (Baez-Estradas \& Ossandón, 2015; Bautista \& Coenders, 2000) y que el AFC corrige gran parte de las carencias de la perspectiva exploratoria, empleando éste como técnica de contraste de los resultados obtenidos en la versión exploratoria (Kline, 2005). Todos los análisis se llevan a cabo con el software IBM SPSS y el subpaquete AMOS v. 22.

\section{Resultados}

\section{Validez de contenido}

La validez de contenido se centra en las manifestaciones realizadas por los expertos seleccionados, considerando que las cuestiones conformantes son las adecuadas y están expresadas de manera clara y relevante; de forma que favorezcan en la mayor medida posible la discriminación entre los sujetos de acuerdo a sus actitudes.

Tras recibir las valoraciones de los 10 evaluadores sobre los tres criterios establecidos, se reciben en los tres aspectos puntuaciones cercanas al valor 4 (mucha): validez (3.8), claridad 
(4) y relevancia (3.9); se establece, en base al juicio de expertos realizado, a pesar del paso del tiempo y los cambios acontecidos en las universidades, el contenido de los ítems es adecuado para nuestra finalidad. Así mismo, no se recibe ningún tipo de consideración significativa sobre la redacción de los ítems, por lo que se decide respetar la estructura y redacción de la versión original.

El nivel de acuerdo interjueces alcanza cotas aceptables, teniendo en cuenta que el coeficiente de variación de las medidas no excede el $20 \%$ (validez $=16 \%$; claridad $=20 \%$; relevancia=18\%). Atendiendo a la validez, claridad y relevancia de cada dimensión por separado, en ningún caso el coeficiente de variación supera el $22 \%$, manteniéndose en un promedio del $17 \%$ de variación.

\section{Fiabilidad}

El cálculo del a de Cronbach arroja un valor aceptable de .75 (Morales, 2000). Este primer indicador muestra que los ítems propuestos son precisos y consistentes a nivel global en su medición. Para llevar a cabo un análisis específico del comportamiento individual de los ítems, se estudia la correlación de cada ítem con respecto a la escala. Con ello se pretende percibir la contribución de cada uno a la explicación de la variabilidad total de la escala. Observando los resultados expuestos en la tabla 1, se observan a nivel general correlaciones ítem-total medias-bajas, y se plantea la posibilidad de suprimir aquellos ítems con valores bajos (<.3). En este caso, el ítem 1 se encuentra en una puntuación límite y es el ítem 3 el que registra una correlación ítem total poco aceptable.

Tabla 1.

Estadísticos total-elemento

\begin{tabular}{lcccc}
\hline \multicolumn{5}{l}{ Valores sin ese elemento. } \\
\hline & Media & Varianza & $\begin{array}{l}\text { Correlac. } \\
\text { elem-total }\end{array}$ & $\begin{array}{l}\boldsymbol{a} \text { de } \\
\text { Cronbach }\end{array}$ \\
\hline Ítem 01. & 51.67 & 23.197 & .294 & .742 \\
\hline Ítem 02. & 51.99 & 21.635 & .420 & .729 \\
\hline Ítem 03. & 52.34 & 22.984 & .149 & .761 \\
\hline Ítem 04. & 51.97 & 21.275 & .434 & .727 \\
\hline Ítem 05. & 52.06 & 20.272 & .517 & .716 \\
\hline Ítem 06. & 51.78 & 21.905 & .467 & .727 \\
\hline Ítem 07. & 51.61 & 23.047 & .450 & .735 \\
\hline Ítem 08. & 52.22 & 22.132 & .301 & .742 \\
\hline Ítem 09. & 51.65 & 23.024 & .420 & .736 \\
\hline Ítem 10. & 52.69 & 20.126 & .385 & .736 \\
\hline Ítem 11. & 52.80 & 20.522 & .352 & .741 \\
\hline Ítem 12. & 52.51 & 19.995 & .550 & .712 \\
\hline Ítem 13. & 52.07 & 22.207 & .343 & .737 \\
\hline & & & &
\end{tabular}

Ante estos valores, si se tratara de una escala de nueva creación, no existiría inconveniente en prescindir de dicha cuestión. Sin embargo, al observar que la eliminación de dicho ítem no mejora considerablemente el estadístico a de Cronbach de la escala, se considera adecuado no prescindir de ninguno de los ítems conformantes. 


\section{Validez de constructo}

En lo referente a la validez de constructo, se aplicó el análisis factorial exploratorio en primer lugar y posteriormente el confirmatorio. A la hora de contrastar las condiciones previas (García et al., 2000), se obtienen los siguientes resultados:

- Prueba de esfericidad de Barlett: $\chi^{2}=421.847 ; p<.001$ (g.l.=78; $a=.05$ ). Se rechaza la hipótesis de esfericidad del espacio multidimensional, por lo que se puede continuar con los análisis.

- Índice $\mathrm{KMO}$ de adecuación muestral. $\mathrm{KMO}=.784$. Esto indica que el valor de las correlaciones entre los pares de variables es elevado en relación con el valor de los coeficientes de correlación parcial existentes (Kaiser, 1974) por lo que se acepta este supuesto previo para continuar con el análisis factorial.

- Por último, se obtienen en todos los casos puntuaciones superiores a .6 en la diagonal principal de la matriz de correlaciones anti-imagen, por lo que se puede garantizar la adecuación muestral de cada una de las variables.

Tabla 2.

Análisis Factorial Exploratorio.

\begin{tabular}{|c|c|c|c|}
\hline & \multicolumn{3}{|c|}{ Componentes } \\
\hline & 1 & 2 & 3 \\
\hline Ítem 01. & .784 & & \\
\hline Ítem 02. & .743 & & \\
\hline Ítem 03. & .520 & & \\
\hline Ítem 04. & .702 & & .329 \\
\hline Ítem 05. & & .445 & .556 \\
\hline Ítem 06. & & & .592 \\
\hline Ítem 07. & & & .622 \\
\hline Ítem 08. & & .401 & .306 \\
\hline Ítem 09. & & & .693 \\
\hline Ítem 10. & & .551 & \\
\hline Ítem 11. & & .783 & \\
\hline Ítem 12. & .447 & .555 & \\
\hline Ítem 13. & & .509 & \\
\hline
\end{tabular}

\begin{tabular}{llll}
\hline de la varianza explicada $=49.32$ & $17.22 \%$ & $16.39 \%$ & $15.71 \%$ \\
\hline
\end{tabular}


En base a los supuestos previos analizados, se cumplen los requisitos para proceder con el AFE, donde se extraen 3 dimensiones y un $49.32 \%$ de la varianza total. La tabla 2 muestra la matriz de componentes rotados (extraídos mediante una rotación ortogonal varimax) y la varianza extraída por cada factor. Se observa cómo, a pesar de obtener pesos factoriales de intensidad moderada en alguno de los ítems, los 3 factores teóricos que se consideraron a la hora del diseño se siguen manifestando de manera nítida en la actualidad.

Tabla 3.

Análisis Factorial Confirmatorio: Índice de ajuste.

\begin{tabular}{ccccccc}
\hline & \multicolumn{3}{c}{ Ajuste global } & \multicolumn{4}{c}{ Ajuste incremental } \\
\hline$\chi^{2}$ & $\chi^{2} /$ g.I & p. & RMSEA & CFI & IFI & NFI \\
\hline 89.365 & 1.441 & .013 & .053 & .923 & .928 & .799 \\
\hline
\end{tabular}

Para confirmar la existencia de los tres factores, se aplica la técnica del AFC mediante la estimación de parámetros por máxima verosimilitud. Una vez aplicado el modelo, se analizan los índices tanto de ajuste tanto global como incremental (tabla 3). En cuanto al ajuste global, el estadístico $\chi^{2}$ alcanza cotas significativas $\left(\chi_{2}=89.37 ; p=.013\right)$ hecho que puede llevar a considerar el ajuste como inadecuado. Sin embargo, este valor puede ser esperable, dado que $\chi 2$ es un estadístico muy sensible al tamaño de la muestra (Hernández-Ramos et al., 2014). Así, ante estas situaciones, es pertinente estudiar otros indicadores de ajuste global como por ejemplo la ratio $\chi$ 2/grados de libertad, o el índice del error de aproximación cuadrático medio (RMSEA). En este caso, ambos índices son aceptables, al obtenerse un valor de la ratio $\chi 2$ /grados de libertad=1.441, inferior a 2 y del estadístico RMSEA $=.053$, inferior a .08, y muy cercano a .05, indicador de buen ajuste (Schreiber et al., 2006).

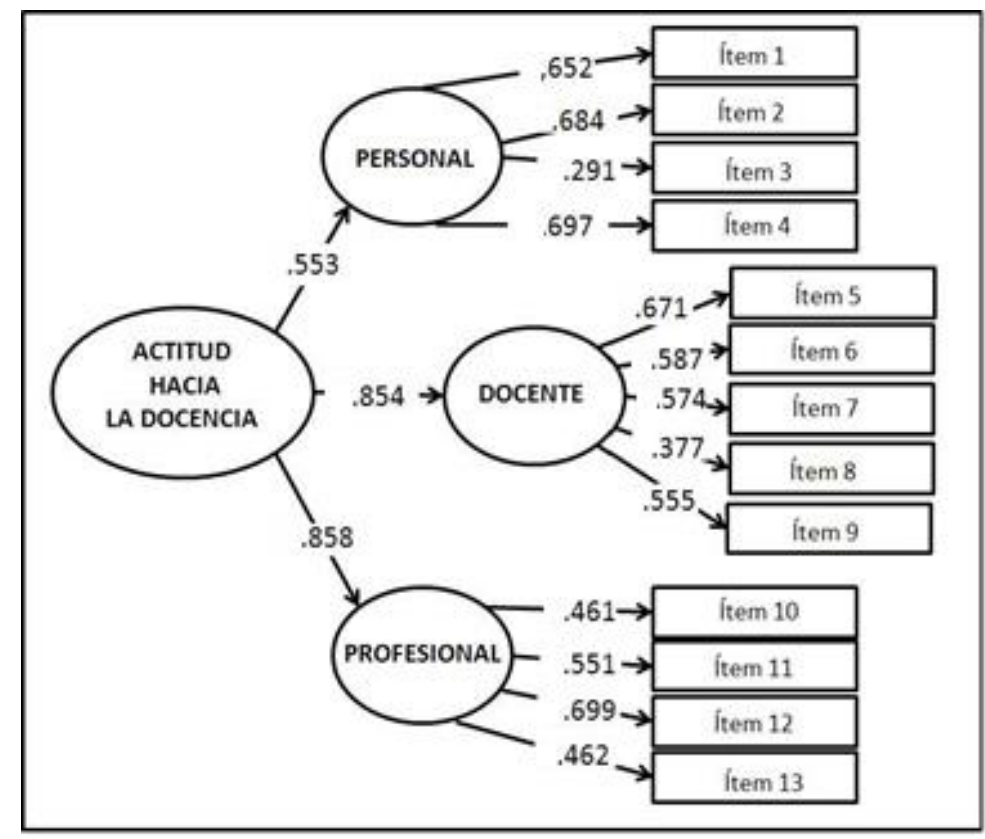

Figura 2. Análisis Factorial Confirmatorio. Solución estandarizada 
En lo referente al ajuste incremental del modelo unifactorial puesto a prueba, el Comparative Fit Index (CFI), índice que compara varios coeficientes de ajuste del modelo, indica un ajuste aceptable (>.9). Por otro lado, el Incremental Fit Index (IFI), también obtiene buenos valores (>.9), incluso superiores al índice CFI. Por su parte, el Indice de Ajuste Normalizado (NFI), que reescala el valor de $\chi 2$ entre 0 y 1 , sigue informando sobre el desajuste observado por este índice.

Por otra parte, en cuanto al peso que cada ítem aporta a cada uno de los factores, como se observa en la figura 2, se localizan contribuciones que, a excepción principalmente del ítem 3 con un índice inferior a .3, son moderadas. Cabe recordar que los estudios de fiabilidad y correlación inter-ítem previos ya apuntaban a esta posibilidad, y que la decisión de mantener el ítem 3 fue justificada por salvaguardar la integridad de la escala original. Así mismo, se debe reseñar cómo los 3 factores no tienen la misma relevancia dentro de la escala completa, destacando un mayor peso de los factores docente (.854) y profesional (.858), frente al personal (.553).

\section{Discusión y conclusiones}

La preocupación por la calidad no puede decirse que sea un tema nuevo en el ámbito de ninguno de los niveles educativos, y desde luego no lo es en el ámbito de la educación superior, siendo este un tema de estudio en todo el mundo (Atrushi \& Woodfield, 2018; Brennan, 2018; Lambert \& Dryer, 2018; Leiber, 2018; Rodríguez-Conde, 2011; Sharma \& Pandher, 2018). Desde hace décadas, es normal encontrarnos con multitud de investigaciones y proyectos diseñados e implementados en pos de la calidad en la enseñanza universitaria. Sin embargo, en infinidad de ocasiones los resultados obtenidos no se asemejan a los deseados y desembocan en modificaciones o cancelaciones. Tradicionalmente se identifica el nivel socioeconómico y cultural, junto a cuestiones como el esfuerzo, la motivación hacia el estudio, la implicación del propio estudiante en su aprendizaje, los apoyos y expectativas familiares, como el predictor más claro del nivel de logro (Márquez \& Madueño, 2016); dejando a un lado aspectos relevantes relacionados con los docentes, como por ejemplo: su actitud.

Se considera que una de las causas que conlleva el fracaso de estos designios reside en que no se suelen tener en cuenta las percepciones y opiniones del profesorado (Covarrubias \& Mendoza, 2016; Gupta et al., 2018), siendo por ello necesaria la existencia de herramientas válidas y fiables que nos ayuden a conocer las actitudes de los docentes (Hernández-Ramos et al., 2016; Toraman et al., 2015).

El estudio presentado muestra una encuesta para evaluar las actitudes autopercibidas del profesorado universitario, en base a tres factores: personal, docente y profesional, hacia su actividad docente. La Escala de Actitud hacia la Docencia fue diseñada a finales de los años 90 en un contexto educativo diferente, y en la actualidad, tras un nuevo proceso de validación de contenido y análisis de las propiedades psicométricas, se puede considerar a este instrumento como válido para su empleo. La escala ha sido validada con éxito tanto a nivel de contenido como a nivel estadístico, mostrando valores aceptables de fiabilidad, entendida como consistencia interna y validez factorial o de constructo, donde se han confirmado los 3 factores teóricos definidos inicialmente. Cabe destacar que, aunque se confirmen los tres factores conformantes de la escala, no todos ellos tienen el mismo peso sobre el constructo. El factor personal, formado por los ítems del 1 al 4, manifiesta una menor repercusión que los factores profesional y docente, formados por los ítems del 5 al 9 y del 10 al 13 respectivamente. Así, como era de esperar, las cuestiones más íntimamente ligados con los aspectos laborales son las que poseen un peso mayor. 
Por tanto, en base a las evidencias obtenidas, se considera que las actitudes del profesorado universitario hacia su rol como docente conforman un constructo que puede medirse mediante la escala propuesta. Resaltando la importancia de considerar la percepción de los docentes a la hora del diseño, el seguimiento y la valoración de las diferentes iniciativas o propuestas que se lleven a cabo en busca de la mejora de la enseñanza universitaria.

Como puntos fuertes y débiles del estudio, se puede resaltar el hecho de que se ofrece una escala simple, concisa y de fácil aplicación, pero que nos ofrece una medición válida y fiable. Además, esta herramienta recoge un alto grado de consolidación teórica al haber sido empleada previamente en otros estudios y validada nuevamente en la actualidad en un contexto formativo dispar, reafirmándose la existencia de una estructura factorial consistente basada en tres dimensiones. Así mismo, también hay que ser conscientes de las limitaciones del instrumento; ya que, a la hora de valorar la fiabilidad de la escala, el coeficiente a de Cronbach influenciado por la concisión de la escala adquiere un valor dentro de la aceptabilidad, pero mejorable.

No obstante, dado que nos desenvolveos en una sociedad dinámica y cambiante como la actual que modifica constantemente la función docente, surgen nuevas líneas de investigación posibles; bien perfeccionando la escala, o empleándola en estudios globales sobre la calidad de la enseñanza universitaria que contemplen las opiniones y actitudes del profesorado. También se abre la posibilidad de replicar este estudio de pilotaje de instrumento con una muestra más amplia que nos ofrezca evidencias más robustas y consolide esta investigación.

\section{Referencias}

Abad, F. J. (2011). Medición en ciencias sociales y de la salud. Síntesis.

Aguilar, M. S. G., \& Cortés, M. E. G. (2016). Free time in the higher education curriculum: Teachers' experiences and opinions. Opcion, 32(Special Issue 7), 707-725.

Atrushi, D. S., \& Woodfield, S. (2018). The quality of higher education in the Kurdistan region of Iraq. British Journal of Middle Eastern Studies, 45(4), 644-659. https://doi.org/10.1080/13530194.2018.1430537

Baez-Estradas, M., \& Ossandón, Y. (2015). Propiedades psicométricas de la escala de presencia social de las mentes en red y su aplicación en UTAmed. Universitas Psychologica, 14(3), 843-853. http://dx.doi.org/10.11144/Javeriana.upsy14-3.ppep

Bautista, J. M., \& Coenders, G. (2000). Modelos de ecuaciones estructurales: Modelos para el análisis de relaciones causales. La Muralla.

Berkant, H. G., \& Derer, Ö. K. (2016). Teachers' opinions regarding the effectiveness of DynEd language education system in english language teaching. Turkish Online Journal of Educational Technology, 2016(DecemberSpeciallssue), 611-620.

Brennan, J. (2018). Success factors of quality management in higher education: Intended and unintended impacts. European Journal of Higher Education, 8(3), 249-257. https://doi.org/10.1080/21568235.2018.1474776

Burgueño, R., Lirola, M.-J., Morales-Ortiz, E., Cueto-Martín, B., \& Medina-Casubón, J. (2018). Análisis psicométrico de la Physical Education Season Survey en estudiantes españoles de educación secundaria. Psychology, Society, \& Education, 10(2), 189-201. https://doi.org/10.25115/psye.v10i2.1093 
Cabero, J., Marín, V., \& Sampedro, B. E. (2018). Technology acceptance model in higher education. Revista de Investigacion Educativa, 36(2), 435-453. https://doi.org/10.6018/rie.36.2.292951

Cáceres, M. T., \& Salas, M. (2012). Valoración del profesorado sobre las competencias genéricas: Su efecto en la docencia. Revista iberoamericana de psicología y salud, 3(2), 195-210.

Çakmak, M. (2011). What are prospective teachers' opinions about their instructors' teaching styles? Procedia - Social and Behavioral Sciences, 15, 1960-1964. https://doi.org/10.1016/i.sbspro.2011.04.035

Caldera, J. F., Reynoso, Ó. U., González, Y. S., \& Zamora, M. R. (2018). Propiedades psicométricas de un instrumento de motivos de ingreso a carreras universitarias de ciencias de la salud. REDU: Revista de Docencia Universitaria, 16(1), 2. https://doi.org/10.4995/redu.2018.6052

Chen, S. (2006). Development of an instrument to assess views on nature of science and attitudes toward teaching science. Science Education, 90(5), 803-819. https://doi.org/10.1002/sce.20147

Covarrubias, C. G., \& Mendoza, M. (2016). Adaptación y validación del cuestionario sentimiento de autoeficacia en una muestra de profesores chilenos. Universitas Psychologica, 15(2), 97-108. https://doi.org/10.11144/Javeriana.upsy15-2.avcs

De Haro, R., Arnaiz, P., \& Nuñez, C. R. (2020). Teacher Competences in Early Childhood Education and Inclusive Education: Design and Validation of a Questionnaire. Revista Electrónica Interuniversitaria de Formación Del Profesorado, 23(1). https://doi.org/10.6018/reifop.407111

Eiroa, F. J., Fernández, I., \& Pérez-Sales, P. (2008). Cuestionarios psicológicos e investigación en Internet: Una revisión de la literatura. Anales de psicología, 24(1), 150-157.

Erwin, P. (2001). Attitudes and Persuasion. Psychology Focus.

Fernández-Batanero, J. M., \& González, J. A. T. (2015). Actitudes docentes y buenas prácticas con TIC del profesorado de Educación Permanente de Adultos en Andalucía. Revista complutense de educación, 33-49. http://dx.doi.org/10.5209/rev_RCED.2015.v26.43812

García, E., Gil, J., \& Rodríguez, G. (2000). Análisis factorial. Muralla.

Grupo Helmántica. (1999). Características profesionales de los docentes universitarios de Castilla y León. Informe de investigación. Junta de Castilla y León.

Gupta, A., Garg, D., \& Kumar, P. (2018). Analysis of students' ratings of teaching quality to understand the role of gender and socio-economic diversity in higher education. IEEE Transactions on Education, 61(4), 319-327. https://doi.org/10.1109/TE.2018.2814599

Gutiérrez, M., Tomás, J.-M., \& Alberola, S. (2018). Teacher support, academic engagement and university student satisfaction. Estudios Sobre Educacion, 35, 535-555. https://doi.org/10.15581/004.34.535-555

Hernández-Ramos, J. P., Herrera-García, E., \& Rodríguez-Conde, M. J. (2017). Influencia del género en la perspectiva del profesorado universitario hacia su función como docente. Universitas Tarraconensis. Revista de Ciències de l'Educació, 1(1), 35-49. https://doi.org/10.17345/ute.2017.1.1751 
Hernández-Ramos, J. P., Martínez-Abad, F., García-Peñalvo, F. J., Herrera-García, E., \& Rodríguez-Conde, M. J. (2014). Teachers' attitude regarding the use of ICT. A factor reliability and validity study. Computers in Humans Behavior, 31, 509-516. https://doi.org/10.1016/j.chb.2013.04.039

Hernández-Ramos, J. P., Martínez-Abad, F., Olmos, S., \& Rodríguez-Conde, M. J. (2016). Evaluación de competencias informacionales con el instrumento IL-HUMASS: Escalamiento multidimensional. Revista iberoamericana de diagnóstico y evaluación psicológica, 42(2), 39-49. http://dx.doi.org/10.21865/RIDEP42_39

Hurtado, A. K. H., \& Madueño, M. L. (2017). Propiedades psicométricas del instrumento prácticas derivadas del aprendizaje social del profesorado. Revista Electrónica Interuniversitaria de Formación del Profesorado, 20(3), 93-106. https://doi.org/10.6018/reifop.20.3.291731

Jiménez, D., Tornel, M., González, J. J., \& Sancho, P. (2019). Perfil del profesorado universitario que emplea un enfoque de enseñanza centrado en el alumnado. Psychology, Society, \& Education, 11(1), 125-135. https://doi.org/10.25115/psye.v1111.2125

Kaiser, H. F. (1974). An index of factorial simplicity. Psychometrika, 39(1), 31-36.

Kerlinger, F., \& Lee, H. (2002). Investigación del comportamiento, Métodos de Investigación en Ciencias Sociales (4. ed.). McGraw-Hill.

Kline, R. B. (2005). Principles and practice of structural equation modeling. Guilford Press.

Lambert, D. C., \& Dryer, R. (2018). Quality of Life of Higher Education Students with Learning Disability Studying Online. International Journal of Disability, Development and Education, 65(4), 393-407. https://doi.org/10.1080/1034912X.2017.1410876

Leiber, T. (2018). Impact evaluation of quality management in higher education: A contribution to sustainable quality development in knowledge societies. European Journal of Higher Education, 8(3), 235-248. https://doi.org/10.1080/21568235.2018.1474775

Maier, M., Greenfield, D., \& Bulotsky-Shearer, R. (2013). Development and validation of a preschool teachers' attitudes and beliefs toward science teaching questionnaire. Early Childhood Research Quarterly, 28(2), 366-378. https://doi.org/10.1016/j.ecresq.2012.09.003

Márquez, L., \& Madueño, M. L. (2016). Propiedades psicométricas de un instrumento para apoyar el proceso de evaluación del docente universitario. Revista Electrónica de Investigación Educativa, 18(2), 53.

Martínez-Clares, P., \& González-Lorente, C. (2019). Competencias personales y participativas vinculantes a la inserción laboral de los universitarios: Validación de una escala. RELIEVE, 25(1). https://doi.org/10.7203/relieve.25.1.13164

Martinez-León, N., Mathes, E., Lucía Avendaño, B., José Peña, J., \& Sierra, J. (2018). Psychometric Study of the Interpersonal Jealousy Scale in Colombian Samples. Revista Latinoamericana de Psicología, 50. https://doi.org/10.14349/rlp.2018.v50.n1.3

Mercado-Salgado, P., Gil-Monte, P., \& Cernas, D. (2016). Validez Inicial de una Escala de Medición del Capital Intelectual en Universidades. Universitas Psychologica, 15(2), 109120. http://dx.doi.org/10.11144/Javeriana.upsy15-2.avcs

Morales, P. (2000). Medición de actitudes en psicología y educación: Construcción de escalas y problemas metodológicos. Universidad Pontificia Comillas. 
Muñoz-Cantero, J.-M., Rebollo-Quintela, N., Mosteiro-García, J., \& Ocampo-Gómez, C.-I. (2019). Validación del cuestionario de atribuciones para la detección de coincidencias en trabajos académicos. RELIEVE - Revista Electrónica de Investigación y Evaluación Educativa, 25(1). https://doi.org/10.7203/relieve.25.1.13599

Naya, M. C., Soneira, C., Mato, M. D., \& Torre, E. de la. (2014). Cuestionario sobre actitudes hacia las matemáticas en futuros maestros de Educación Primaria. Revista de Estudios e Investigación en Psicología y Educación, 1(2), 141-149. https://doi.org/10.17979/reipe.2014.1.2.11

Ozturk, M. A. (2010). An Exploratory Study on Measuring Educators' Attitudes toward Educational Research. Educational Research and Reviews, 5(12), 758-769.

Prieto, G. (2012). Medición en Ciencias Sociales y de la Salud. Psicothema, 24(1).

Rodríguez-Conde, M. J. (2011). La garantía de la calidad, base de la movilidad. REDU: Revista de Docencia Universitaria, 9(3), 99-117. https://doi.org/10.4995/redu.2011.6151

Rodríguez-Conde, M. J., Olmos, S., \& Martínez-Abad, F. (2012). Propiedades métricas y estructura dimensional de la adaptación española de una escala de evaluación de competencia informacional autopercibida (IL-HUMASS). Revista de investigación educativa, RIE, 30(2), 347-365. https://doi.org/10.6018/rie.30.2.120231

Sales, A., García-López, R., Ferrández, M. R., \& Moliner, M. O. (2006). Elaboración de instrumentos de medida de las actitudes y opiniones del profesorado universitario. Relieve: Revista ELectrónica de Investigación y EValuación Educativa, 12(1), 8-16.

Schreiber, J. B., Nora, A., Stage, F. K., Barlow, E. A., \& King, J. (2006). Reporting Structural Equation Modeling and Confirmatory Factor Analysis Results: A Review. Journal of Educational Research, 99(6), 323-337. https://doi.org/10.3200/JOER.99.6.323-338

Sharma, P., \& Pandher, J. S. (2018). Quality of teachers in technical higher education institutions in India. Higher Education, Skills and Work-based Learning, 8(4), 511-526. https://doi.org/10.1108/HESWBL-10-2017-0080

Sicilia, Á., Alcaraz-lbañez, M., Lirola, M.-J., \& Burgueño, R. (2017). Propiedades psicométricas de la versión española del Cuestionario de Contenido de Metas en el Ejercicio. Revista Latinoamericana de Psicología, 182-193. https://doi.org/10.1016/j.rlp.2016.10.001

Soriano, A. M. (2014). Diseño y validación de instrumentos de medición. Editorial Universidad Don Bosco, 8(13), 19-40. https://doi.org/10.5377/dialogos.voi14.2202

Toraman, C., Acar, F., \& Aydin, H. (2015). Primary School Teachers' Attitudes and Knowledge Levels on Democracy and Multicultural Education: A Scale Development Study. Revista de Cercetare si Interventie Sociala, 49, 41-58.

Urbano-Contreras, A., Iglesias-García, M. T., \& Martínez-González, R. A. (2019). Diseño y validación de la Escala de Tiempo Compartido en la Pareja (TCP). Psychology, Society, \& Education, 11(2), 165-175. https://doi.org/10.25115/psye.v11i2.1909

Zych, I., Ortega-Ruiz, R., Muñoz-Morales, R., \& Llorent, V. J. (2018). Dimensions and Psychometric Properties of the Social and Emotional Competencies Questionnaire (SEC-Q) in youth and adolescents. Revista Latinoamericana de Psicología, 50(2), 98106. https://doi.org/10.14349/rlp.2018.v50.n2.3 